\title{
Role of Membrane Potential in Protein Folding and Domain Formation During Secretion in Escherichia coli
}

\author{
Bruce R. Copeland, Robert Landick, Penelope M. Nazos, and \\ Dale L. Oxender \\ Department of Biological Chemistry, University of Michigan, Ann Arbor, Michigan \\ 48109
}

The synthesis and processing of the periplasmic components of the leucine transport system of $\mathrm{E}$ coli have been studied to determine the role played by transmembrane potential in protein secretion. Both the leucine-isoleucine-valine binding protein and the leucine-specific binding protein are synthesized as precursors with 23 amino acid $\mathrm{N}$-terminal leader sequences. The processing of these precursors is sensitive to the transmembrane potential. Since the amino acid sequence and the crystal structure have been determined for the leucine-isoleucine-valine binding protein, it and the closely related leucine-specific binding protein represent convenient models in which to examine the mechanism of protein secretion in $\mathrm{E}$ coli. A model for secretion has been proposed, suggesting a role for transmembrane potential. In this model, the $\mathrm{N}$-terminal amino acid sequence of the precursor is assumed to form a hairpin of two helices. The membrane potential may orient this structure to make it accessible to processing. In addition, the model suggests that a negatively charged, folded domain of the secretory protein may electrophorese toward the trans-positive side of the membrane, thus providing an additional role for the transmembrane potential.

Key words: bacterial protein secretion, transmembrane potential, secondary structure prediction, protein folding, electric field, domain formation, binding proteins, periplasmic

Leucine-specific binding protein (LS-BP) and leucine-isoleucine-valine binding protein (LIV-BP) are periplasmic proteins of $E$ coli that function in the transport of leucine into the cell [1]. Both proteins are initially synthesized in the cytoplasm as

\footnotetext{
Abbreviations used: LS-BP, leucine-specific binding protein; LIV-BP, leucine-isoleucine-valine binding protein; CCCP, carbonyl cyanide m-chlorophenylhydrazone.
}

Robert Landick's present address is Biological Sciences, Stanford University, Stanford, CA 94305.

Received October 4, 1983; revised and accepted January 13, 1984.

(C) 1984 Alan R. Liss, Inc. 
precursors with 23 amino acid leader sequences that are removed during export across the inner membrane to the periplasmic space [2-4]. The genes for these proteins have been cloned on plasmids. In addition, the DNA sequence for LIV-BP and LS-BP [5], the amino acid sequence for LIV-BP [6], and a preliminary crystal structure for LIVBP [7] are available. Extensive experimental studies have been made of the export process for these proteins in vitro $[3,4,8]$ and in vivo [9-11]. LIV-BP and LS-BP, therefore, represent convenient model systems that are available for the study of protein secretion in bacteria.

Precursors of periplasmic binding proteins have been shown to be synthesized on membrane-bound polysomes [12]. In vitro experiments indicate that the processing of the leucine binding protein precursors is associated with the inner membrane of $\mathrm{E}$ coli [3], and the leader peptidase, isolated by Wickner and coworkers [13,14], appears to process these binding proteins [8]. The processing can be demonstrated to occur posttranslationally in vivo $[9,10]$ and in vitro [3]. Processing of LIV-BP and LS-BP is inhibited by ionophores such as valinomycin and carbonyl cyanide $\mathrm{m}$-chlorophenylhydrazone (CCCP) under conditions where these agents collapse the transmembrane potential $[9,10]$. (Similar results have been obtained with other secreted proteins of $E$ coli [15-18].) The resulting precursors for LIV-BP and LS-BP accumulate and can be isolated with the inner membrane of $\mathrm{E}$ coli, suggesting that these precursors are associated with the membrane even in the absence of a transmembrane potential. These results indicate that the transmembrane potential is either required for or greatly facilitates processing of LIV-BP and LS-BP in vivo. It has not been possible to demonstrate any requirement for transmembrane potential in the in vitro reconstitution of processing activity in membranes [19,20] [Copeland and Oxender, unpublished].

Specific secretion proteins have been found to be involved in eukaryotic protein secretion [21-23], and some evidence has accumulated to indicate that certain additional proteins are involved in bacterial protein secretion [24-27]. We have recently demonstrated that processing of LS-BP is inhibited by the secA mutation characterized by Oliver and Beckwith [26,27], although this mutation appears to have less effect on LS-BP than on other secreted proteins described by Oliver and Beckwith [26]. The role of these specific proteins in the secretory process remains to be determined. It is clear, however, that processing and other exportlike events can be reconstituted in vitro in liposome vesicles under conditions where only soluble $\mathrm{E}$ coli proteins are present in addition to the leader peptidase [19,20] [Copeland and Oxender, unpublished].

The detailed molecular steps involved in the translocation and processing steps of protein secretion are not well understood. Early models for the protein secretion process emphasized the concept of extrusion of nascent protein chains through the membrane, coupled to elongation of the chains during protein synthesis. Such a process would be necessarily cotranslational, regardless of whether the actual processing of the leader sequence was cotranslational or posttranslational. It is difficult to suggest a role for membrane potential in such models. Indeed, the extrusion of the positively charged $\mathrm{N}$-terminus of the leader sequence through the membrane would be energetically uphill in the presence of a trans-positive membrane potential difference. Moreover, it is difficult to rationalize nascent chain extrusion models with the observation that processing and vectorial translocation can occur in vitro when membrane vesicle preparations are added posttranslationally [3]. Finally, recent ex- 
periments by Randall [28] indicate that the maltose binding protein and the ribose binding protein do not become accessible to protease on the outer side of the membrane until the proteins have been nearly completely synthesized. It has been estimated that nascent chain extrusion models would predict sensitivity to protease within 50 to 100 residues after the start of synthesis [28].

More recent models for extrusion of nascent chains allow the positive charge near the $\mathrm{N}$-terminus to anchor the leader sequence on the cytoplasmic side of the membrane, facilitating the formation of a hairpin structure with extrusion of the mature part of the protein through the membrane as a nascent chain $[29,30]$. This type of loop model could be used to explain the involvement of membrane potential, as will be discussed in a slightly different context later. A hydrophilic protein pore would probably be required for such a process of nascent chain extrusion, since the energy associated with inserting uncompensated charges directly into even a fairly polar membrane is highly unfavorable (see, for example, [31]).

Alternatives to nascent chain extrusion models emphasize either movement of completely folded proteins across the membrane (clearly posttranslational) or movement of specific portions of the incompletely folded proteins across the membrane (cotranslational or posttranslational). The membrane trigger hypothesis [32] is an example of a model that incorporates the concept of conformational changes during protein secretion. Since many proteins are exported cotranslationally and it is possible to force cotranslational export of some proteins that are normally exported posttranslationally, it is likely that translocation of limited domains of proteins and not translocation of the entire folded protein occurs. Although much work has been done to identify critical structural elements in leader sequences, it is likely that folding of additional domains of precursor proteins will also be important in the secretory process. Consideration of domain formation may be important for understanding the mechanism of protein secretion in general, as well as for understanding the role of transmembrane potential. This report will examine some of the steps in the secretion of LIV-BP and LS-BP in terms of protein folding, domain formation, and possible roles played by transmembrane potential.

\section{STRUCTURAL PROPERTIES OF LIV-BP AND LS-BP}

In order to facilitate discussion of the possible role of domains and protein folding in the secretion of the leucine binding proteins, it is useful to consider some of the sequence and structural properties of the binding proteins and their precursors. Figure 1 shows the amino acid sequences derived from the DNA sequences for the $\mathrm{N}$-terminal portions of the precursors of LIV-BP and LS-BP [5]. Also shown in Figure 1 are the positions of the processing sites determined by comparison between amino acid sequence data for the mature proteins $[3,6]$ and the amino acid sequences derived from DNA sequences for the precursors. The leader sequences for both binding proteins are 23 amino acids in length. The mature LS-BP contains 346 amino acids, and the mature LIV-BP contains 344 amino acids. The degree of amino acid sequence homology between the two mature proteins is $80 \%$. The leader sequences themselves have only $40 \%$ sequence homology, but they share a number of structural features, which are also frequently found in leader sequences for other exported proteins in $\mathrm{E}$ coli. These features are (1) the presence of two or more positively charged residues near the $\mathrm{N}$-terminus of the leader sequence; (2) the existence of a 


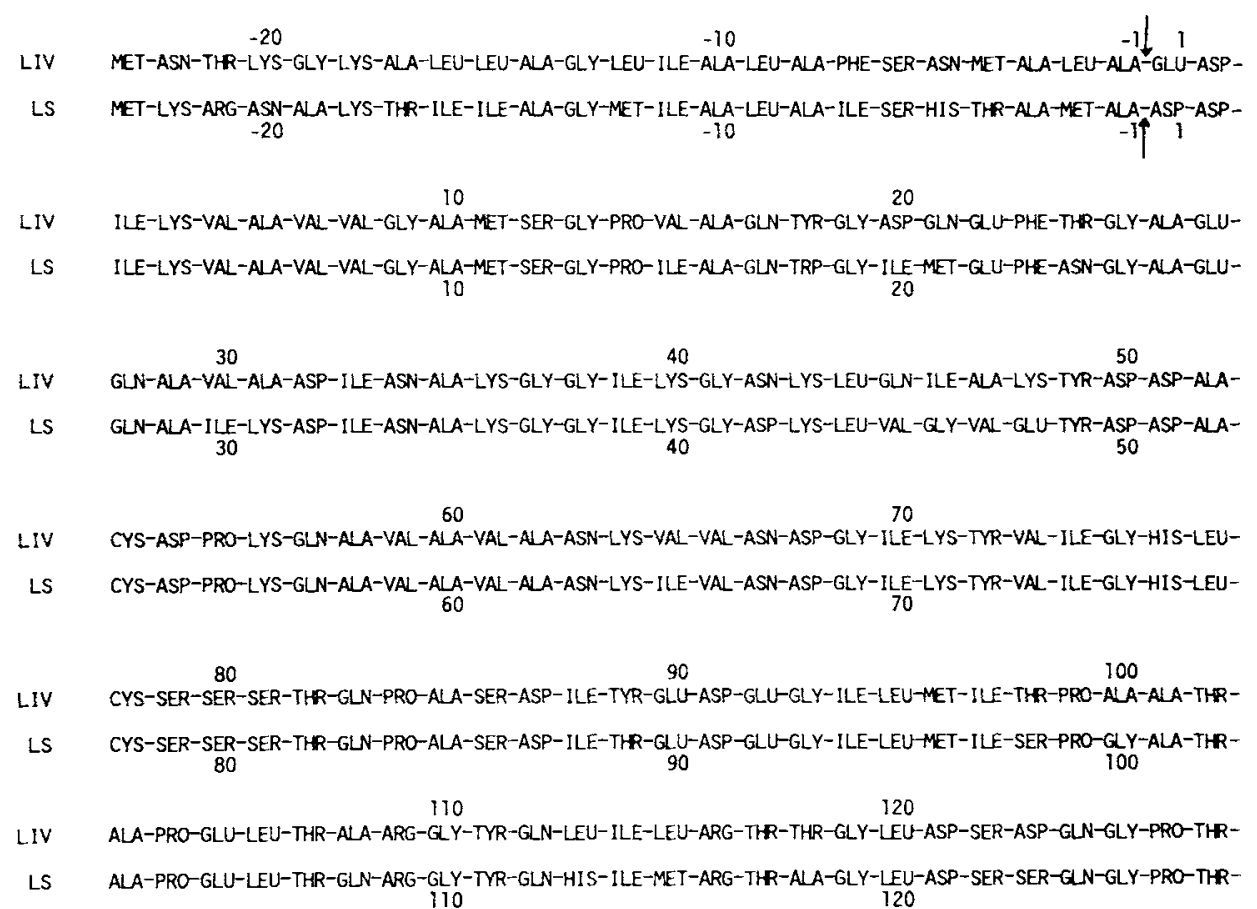

Fig. 1. Amino acid sequences obtained from DNA sequences [5] for the $N$-terminal portions of the precursors of LIV-BP and LS-BP. Amino acid sequence data for the mature forms of these proteins $[3,6]$ were compared with these sequences to determine the locations of the processing sites. These sites are indicated by arrows.

core region consisting of hydrophobic amino acids, which is believed to be a helical structure in the membrane; and (3) the presence of several negatively charged or polar amino acids at or just past the processing site.

A preliminary crystal structure has been obtained by Saper and Quiocho for the mature LIV-BP [7]. A stereo picture of the $\alpha$-carbon backbone deduced from this structure is reproduced in Figure 2A. LIV-BP, like other periplasmic binding proteins that have been studied by crystallography, is a bilobate protein consisting predominantly of alternating sections of $\alpha$-helix and $\beta$-sheet. The pattern and course of these domains is indicated conceptually in Figure $2 \mathrm{~B}$. The bulk of the upper lobe is formed from the first five $\beta$-sheets and first four helix regions. The bulk of the lower lobe is formed from the next five $\beta$-sheet and five helix regions. The $\alpha$-carbon backbone then wanders back to the upper lobe to add two more helix regions and finally returns to the lower lobe with two $\beta$-sheet regions near the $\mathrm{C}$-terminus.

In the following discussion it should be remembered that the $\alpha$-carbon backbone in Figure $2 \mathrm{~A}$ is a preliminary structure deduced from a $3-\AA$ resolution crystal structure, and there may be some ambiguity about the locations of all of the amino acids, particularly at the two ends of the protein and also in the turns between regions of secondary structure.

In order to better define the relationship between the amino acid sequence and the structure in Figure 2, we used the method of Chou and Fasman [33] to predict 
secondary structure from the amino acid sequence. We then compared these predictions with the order and approximate lengths of the $\alpha$-helix and $\beta$-sheet regions in Figure 2A. Figure 3 shows the amino acid sequence for the first 125 amino acids of the mature LIV-BP, along with the positions of probable $\alpha$-helix, $\beta$-sheet, and $\beta$-turn that were determined from calculations using the Chou-Fasman method. Using the rules formulated by Chou and Fasman for distinguishing between $\alpha$-helix, $\beta$-sheet, and $\beta$-turn [33], we have predicted the locations of the four helical domains and five $\beta$-sheet domains that make up the upper lobe of LIV-BP. For the most part, the lengths of these predicted regions of secondary structure, the spacing between them, and the positions of these regions along the amino acid sequences are in good agreement with the structure in Figure $2 \mathrm{~A}$. The five predicted $\beta$-sheet regions in Figure 3 all have higher mean $\beta$-sheet probabilities than $\alpha$-helix or $\beta$-turn probabilities. The four predicted $\alpha$-helix regions in Figure 3 have higher mean $\alpha$-helix probability than $\beta$-sheet or $\beta$-turn probability, except for the first half of helix III, which we suggest may be a $3_{10}$ helix composed of linked $\beta$-turns. We note that this third helix is rather distorted in the stereo picture in Figure 2. All other possible positionings of alternating $\beta$-sheet and $\alpha$-helix along the amino acid sequence have lower probabilities than the one in Figure 3.

The high degree of homology noted earlier between sequences for mature LIVBP and mature LS-BP extend throughout the lengths of the proteins. The secondary structure for LS-BP predicted using the Chou-Fasman method is in correspondingly good agreement with the predictions in Figure 3 for LIV-BP (data not shown). The predicted secondary structures make it possible to position the charged residues in the amino acid sequences along the structures of the proteins. These charged residues are indicated $(+$ and -$)$ along the sequence in Figure 3 for the upper lobe of LIV-BP. (In this description we have designated the histidine residues as having positively charged residues at neutral $\mathrm{pH}$.) We are now in a position to use our understanding of the structure of LIV-BP and LS-BP to discuss protein folding and domain formation in secretion.

\section{DOMAIN FORMATION DURING EXPORT OF LEUCINE BINDING PROTEINS}

One structural element that has been an important feature in many models of protein secretion is a hairpin formed between the leader sequence and the mature protein $[29,30,32,34,35]$. As previously mentioned, the presence of a positive charge at the $\mathrm{N}$-terminus makes translocation of the entire leader sequence across the membrane energetically unfavorable against a trans-positive electric field. Furthermore, such a process does not provide an explanation for possible involvement of transmembrane potential in secretion. The alternative model in which the $\mathrm{N}$-terminus of the leader sequence is located on the cytoplasmic side of the inner membrane and the processing site on the outer side confers a stabilization of $1-5 \mathrm{kcal} / \mathrm{mol}$ in the presence of a typical trans-positive electric field and involves, at least initially, the formation of a hairpin structure. Such a transmembrane conformation is also consistent with secondary structure predictions that the hydrophobic core of a leader sequence forms an $\alpha$-helix. Helical structures can be quite stable in membrane hydrocarbon environments by virtue of their placement of hydrogen bonds in the interior of the helix and amino acid side chain residues (in this case hydrophobic) on 

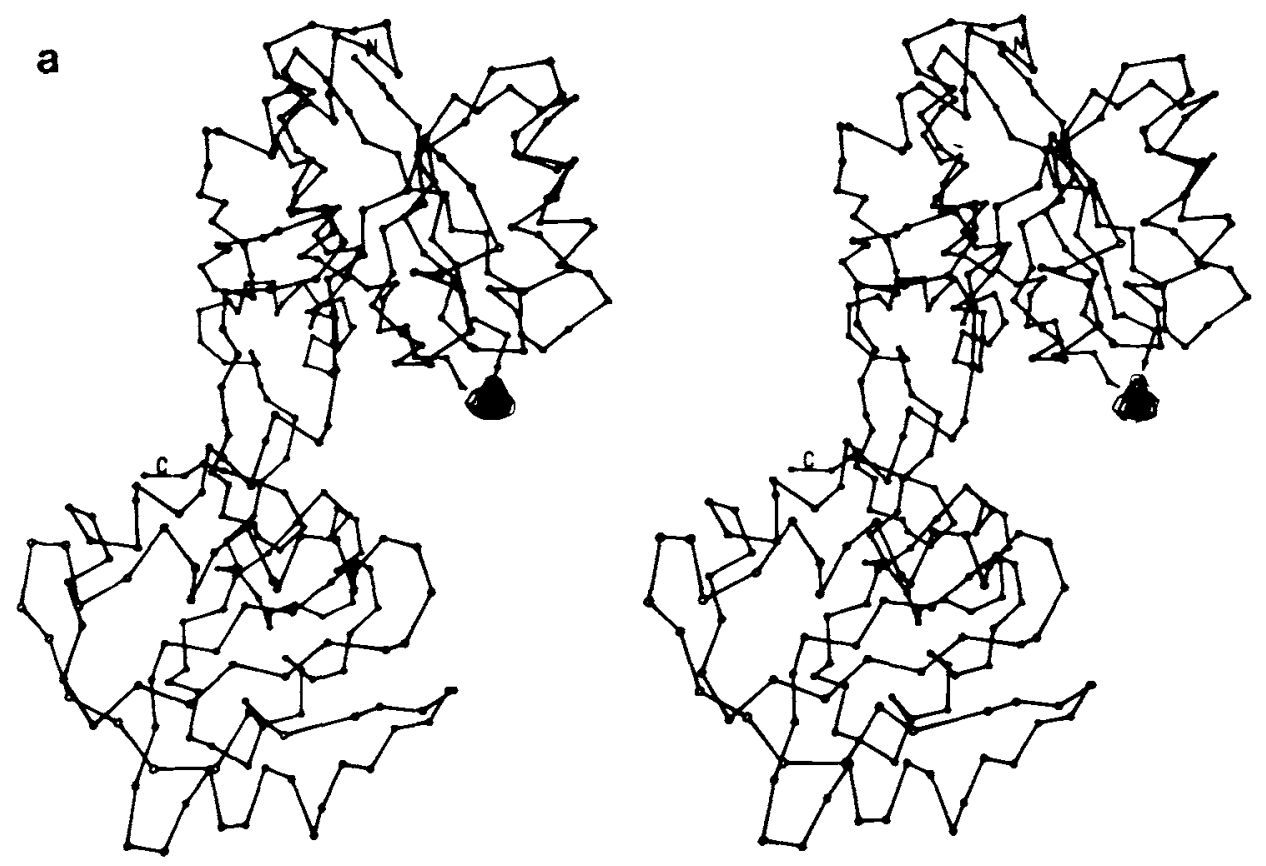

Fig. 2. Three-dimensional structure for mature LIV-BP. a) Stereo picture of the $\alpha$-carbon backbone (reproduced with permission from Saper and Quiocho [7l). b) Schematic representation of the domains of secondary structure observed in LIV-BP. The dark spot between the two lobes of LIV-BP in a) indicates the location of ligand doped into the ligand-free crystal. Helical regions in $b$ ) are numbered with Roman numerals, and regions of $\beta$-sheet are lettered in order of occurrence from the N-terminus.

the outside of the helix. Secondary structure predictions by the Chou-Fasman method [33] for the LIV-BP and LS-BP leader sequences give very high probabilities for $\alpha$ helix formation over most of the length of the leader sequences.

Figure 4 shows a specific model for a helical hairpin structure in secretion of the leucine binding proteins. In proposing this model, we have taken advantage of the observation that not only do the leader sequences themselves have high probability for helix formation, but the first 10 to 33 amino acids in the mature part of the protein are also predicted to have long sections with $\alpha$-helix forming probability, as indicated in Figure 3. Although part of this region is predicted to have higher probability for $\beta$-sheet than for $\alpha$-helix using the Chou-Fasman method, we expect that the $\alpha$-helix structure will be preferred in a hydrophobic membrane environment. Such a hairpin of two helical sections near the $\mathrm{N}$-terminus is, therefore, a reasonable possibility in a secretion model for LIV-BP and LS-BP.

A major stabilization for the hairpin structure shown in Figure 4 can be expected from dipolar interactions. Protein helices have dipole moments that result from the polarization in hydrogen bonds. The strength of the resulting favorable dipolar interaction between two antiparallel helices has been estimated to be in the range of 8-25 $\mathrm{kcal} / \mathrm{mol}$, depending on lengths of the helices and distance of their separation [36]. For the model in Figure 4, we may expect the interaction between the two 


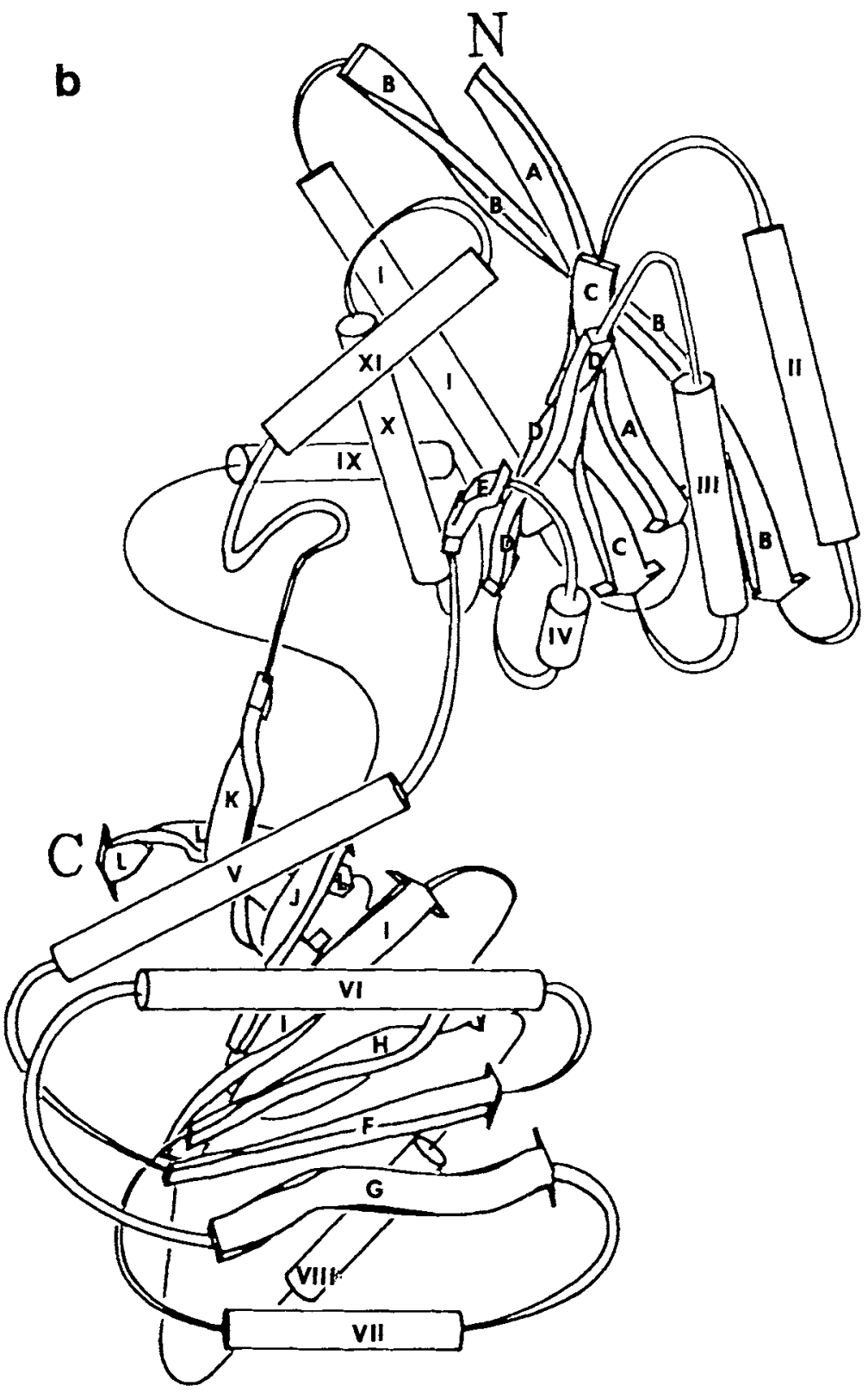

Fig. 2 (continued)

helices in the hairpin to be stronger than this, since there is an additional contribution to the dipole moment of the leader sequence that results from the positively and negatively charged amino acid residues at the two ends of the leader sequence. Thus the overall conception in the first two steps of Figure 4 is that the strong local electric field from the leader sequence dipole stabilizes a hairpin structure in which one or more subsequent helical region(s) runs antiparallel to the leader sequence. The resulting hairpin structure, however, retains a significant net dipole, which is aligned 


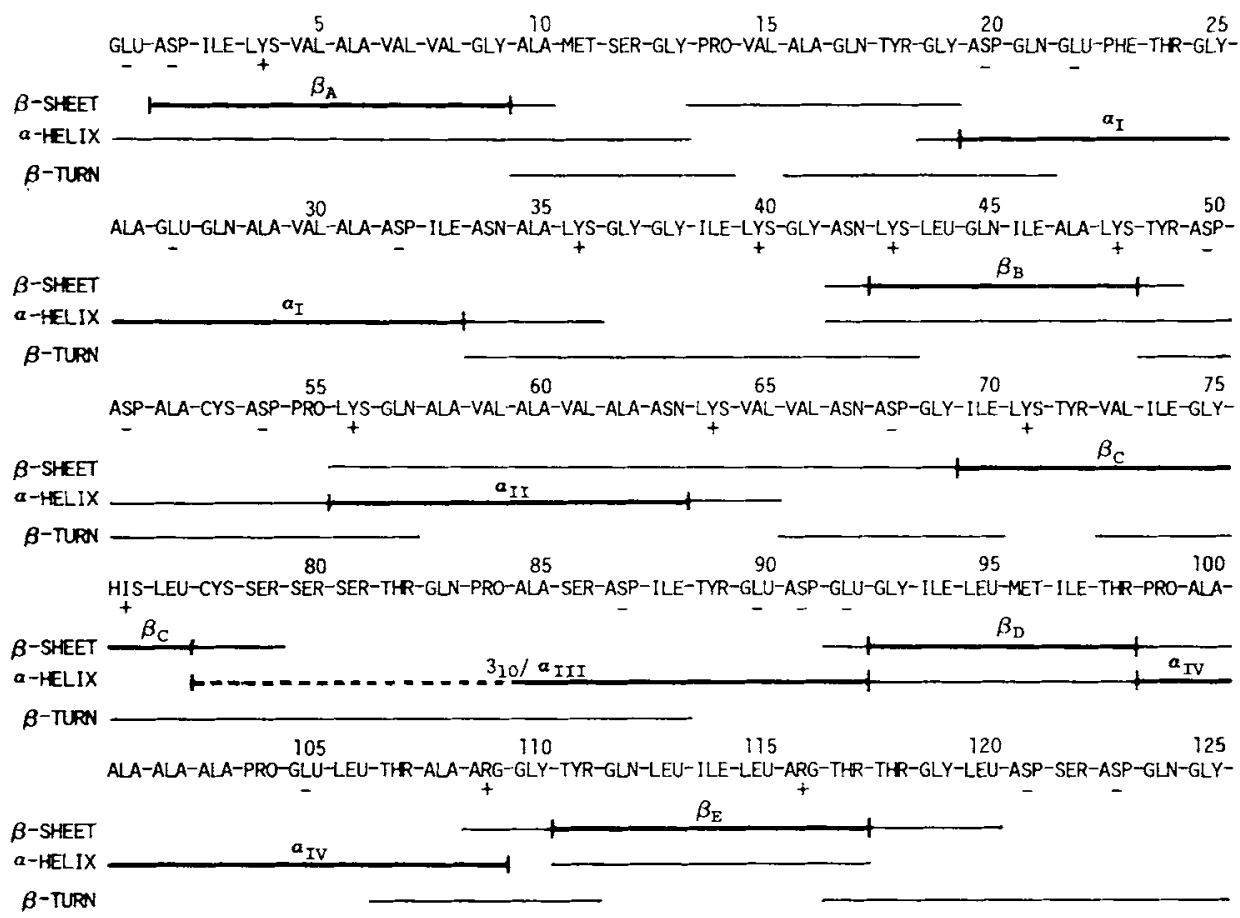

Fig. 3. Secondary structure predictions for the upper lobe of mature LIV-BP. The first 125 amino acids of LIV-BP are shown, along with lines indicating regions, respectively, of probable $\beta$-sheet, $\alpha$-helix, and $\beta$-turn. These regions were identified using the numerical methods of Chou and Fasman [33]; for $\beta$ sheet and $\alpha$-helix, they represent contiguous sections of tetrapeptides that have tetrapeptide mean conformational parameters greater than 1.00 and sufficient sheet-or helix-forming residues to nucleate these respective structures; for $\beta$-turns they represent contiguous sections of tetrapeptides that have tetrapeptide mean conformational parameters greater than 1.00 and tetrapeptide frequencies greater than $7.5 \times 10^{-5}$. The bracketed bold lines indicate regions that are predicted to correspond to the first five $\beta$-sheet and first four helix regions in mature LIV-BP. These regions were predicted using the probability hierarchy described by Chou and Fasman [33] for distinguishing between $\beta$-sheet, $\alpha$-helix, and $\beta$-turn. In two instances, $\beta_{\mathrm{B}}$ and $\alpha_{\mathrm{II}}$, there is nearly equal probability that the region could be an alternative structure. The region indicated as $\alpha_{\text {III }}$ contains a predicted region of $\alpha$-helix considerably shorter than the observed helical region in the three-dimensional structure; however, there is a long region of overlapping predicted $\beta$-turn. We suggest that helix III may consist of a $3_{10}$ helix formed from linked $\beta$ turns that merge with $\alpha$-helix. Charged amino acids are indicated $(+$ and -$)$ under the amino acid abbreviations in the sequence.

in a transmembrane orientation by the electric field associated with the trans-positive membrane potential difference.

As elaborated in the previous discussion, the electric field across the inner membrane may stabilize the helical hairpin in a transmembrane conformation, which facilitates removal of the leader sequence through proteolytic processing by the leader peptidase. Once the leader sequence is removed, the remainder of the protein is no longer stable in the membrane. As shown in Figure 4, the protein therefore refolds into the periplasmic space with the central strand of $\beta$-sheet in the $\mathrm{N}$-terminal domain being formed from the remaining half of the hairpin, which was an $\alpha$-helix in the 


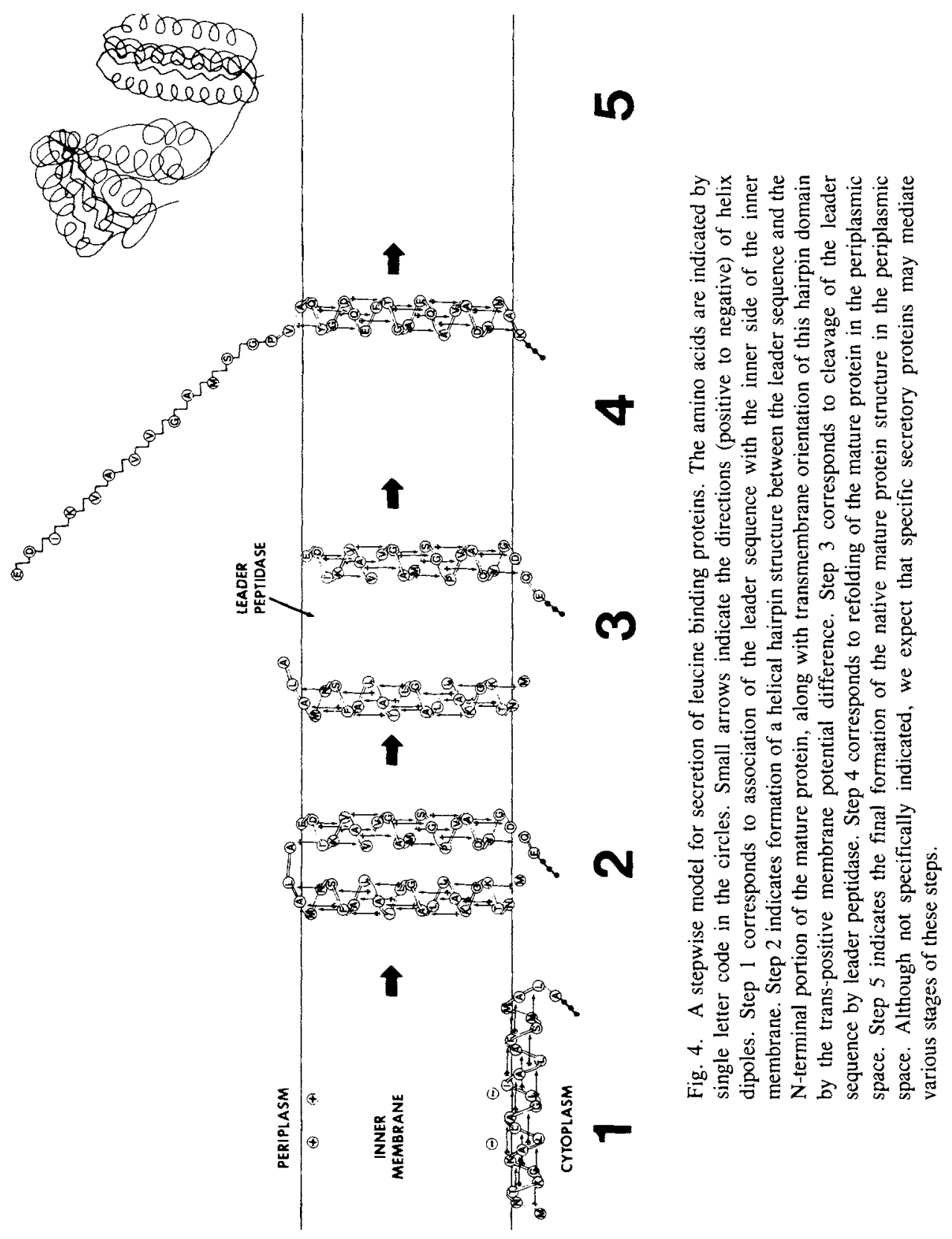


membrane. During the entire process of translocation across the membrane, folding and refolding of additional parts of the protein may also be critical. We expect that this will be energetically assisted by a combination of protein folding energy and possibly also by other interactions with the electric field in the membrane, as discussed below.

\section{CAN TRANSMEMBRANE POTENTIAL BE USED TO "ELECTROPHORESE", NEGATIVELY CHARGED DOMAINS OF PROTEIN ACROSS THE MEMBRANE?}

Recent studies by Randall [28] suggest that substantial portions of maltose binding protein and ribose binding protein precursors must be synthesized before these proteins become proteolytically sensitive on the outer side of the inner membrane. Recent experiments in our laboratory with mutant forms of LS-BP [11] indicate that significant portions of the $\mathrm{C}$-terminus are not required for secretion. Taken together, these results suggest that some substantial $\mathrm{N}$-terminal part or domain of a precursor may have to be translocated across the membrane before leader peptidase can cleave the leader sequence. It is therefore worthwhile to consider what factors might influence the folding and translocation of such a domain of a precursor. In the case of the leucine binding proteins, considerable negative charge density exists in the $\mathrm{N}$-terminal portion of the mature proteins (six net negative charges in the first 108 amino acids of mature LIV-BP and seven negative charges in the corresponding part of LS-BP). The electric field of approximately $10^{5} \mathrm{~V} / \mathrm{cm}$ associated with a typical trans-positive membrane potential should be sufficient to move or "electrophorese" domains of protein secondary structure that contain net negative charge. One role for transmembrane potential might therefore be to electrophorese negatively charged, folded domains of the leucine binding proteins across the membrane. We envisage that electrophoresis of a protein domain may involve the interaction of the forces of protein folding and electrophoretic migration so that a protein domain present on the inside of the membrane at a higher free energy level moves across the membrane in response to the electric field and ultimately refolds into a new conformation at a lower free energy level on the outside of the membrane. Interestingly, the lengths of sequence that could be involved in the formation of negative domains of leucine binding proteins are sufficient to form most of the upper lobe of the mature proteins. In this model we assume that, after the leader sequences are removed, the remaining lengths of sequence spontaneously refold to form the upper lobe of the mature proteins in the aqueous phase.

It is important to recognize that the electric field that might electrophorese a negative domain of a protein across the membrane is localized predominantly in the hydrophobic part of the membrane. Thus, in order for a charged domain to be electrophoresed by the electric field, it must first reach the hydrophobic part of the membrane where the field is located. There are several energetic factors that will influence the likelihood of reaching the hydrophobic region. Movement of an unneutralized charge from a polarizable (high dielectric) environment to a nonpolarizable (low dielectric) environment is unfavorable because the electron density is no longer spread over such a large volume. An upper limit for this unfavorable energy can be estimated from the difference between the Born energy (see, for example, [37]) for a charge in a low dielectric medium (membrane) and the Born energy for the same 
charge in a high dielectric medium (water). We calculate this to be $7.3 \mathrm{kcal} / \mathrm{mol}$ for a single charge of mean radius $2 \AA$ going from water $(\epsilon=80)$ to an environment of $\epsilon=10$, or $40 \mathrm{kcal} / \mathrm{mol}$ for the same ion going to an environment of $\epsilon=2$. There is a favorable energy associated with moving most amino acid residues (apart from the charge itself, which was just treated) from a polar to a hydrophobic environmentparticularly if hydrogen bonds between residues are retained [38]. For example, if we take the exposed surface of an $\alpha$-helix to be $29 \AA^{2} /$ amino acid residue, and use Chothia's value of $0.024 \mathrm{kcal} / \AA^{2}$ hydrophobic stabilization energy [38], we calculate a stabilization of $0.67 \mathrm{kcal} / \mathrm{mol}$ amino acid residue of helix in a hydrophobic environment. If the exposed surface area is $58 \AA^{2} /$ residue, then the corresponding stabilization is $1.3 \mathrm{kcal} / \mathrm{mol} / \mathrm{residue}$. Thus we conclude that, for transfer into a hydrophobic environment, the hydrophobic stabilization for amino acid residues that are located in domains of secondary structure may be of sufficient magnitude to offset the unfavorable energy associated with transfer of unneutralized charges, depending on the lengths of the domains and the number of charges. This means that penetration of some charged domains of proteins into the hydrophobic portion of a membrane is permitted, and under these conditions it would be possible for domains to electrophorese across the membrane. The folding or conformation of these domains in the membrane may be somewhat different than that found in aqueous solution. Indeed, the ability to form more than one stable conformation could provide stability for the charged domain within the hydrophobic membrane.

The model we have presented in Figure 4 is, therefore, consistent with two possible roles for transmembrane potential in secretion of leucine binding proteins. One role would be to stabilize a transmembrane orientation of a helical hairpin structure that contains the leader sequence, as described above. An additional role may be to electrophorese negative $\mathrm{N}$-terminal domains of the precursors across the membrane. It was our experimental results with the leucine binding proteins that lead us to focus attention on the translocation of early domains during protein secretion. We do not wish to suggest that either of these roles will be universally applicable to secreted proteins. The possibility that this model can be applied to other secretory proteins remains to be established. With respect to electrophoresis of negative domains, it should be noted that the vast majority of secreted proteins in $\mathrm{E}$ coli have isoelectric points lower than neutral $\mathrm{pH}$ and would therefore be expected to have domains of net negative charge under physiological conditions. It is important to recognize, however, that in the proposed model it is the net charge on a domain in the N-terminal region and not necessarily the overall isoelectric point of the protein that determines whether electrophoresis can play a role in translocation and secretion of the protein.

\section{ACKNOWLEDGMENTS}

This work was supported in part by grant GM11024 from the National Institutes of Health. B. Copeland held an Arthur F. Thurnau Postdoctoral Fellowship and P. Nazos was supported by an American Association of University Women's Fellowship.

We would like to thank Dr. Shaun Black for making the secondary structure prediction program CHOFAS available to us. We are grateful to Dr Florante Quiocho for early communication of the three-dimensional structure for LIV-BP. Dr. Steve Quay has provided helpful discussions. 


\section{6:JCB Copeland et al}

\section{REFERENCES}

1. Landick R, Oxender DL: In Martonosi A (ed): "Membranes and Transport," Vol. 2. New York: Plenum Press, 1982, pp 81-91.

2. Oxender DL, Anderson JJ, Daniels CJ, Landick R, Gunsalus RP, Zurawski G, Selker E, Yanofsky C: Proc Natl Acad Sci USA 77:1412-1416, 1980.

3. Daniels CJ, Anderson JJ, Landick R, Oxender DL: J Supramol Struct 14:305-311, 1980.

4. Oxender DL, Anderson JJ, Daniels CJ, Landick R, Gunsalus RP, Zurawski G, Yanofsky C: Proc Natl Acad Sci USA 77:2005-2009, 1980.

5. Landick R: Ph.D. Thesis "The LIV-I Transport Gene Cluster of Escherichia coli," University of Michigan, Ann Arbor, Michigan, 1983.

6. Ovchinnikov YA, Aldanova NA, Grinkevich VA, Arzamazova NM, Moroz IN, Nazimov IV: FEBS Lett 78:313-316, 1977.

7. Saper MA, Quiocho FA: J Biol Chem 258:11057-11062, 1983

8. Copeland BR, Su TZ, Landick RC, Nazos PM, Oxender DL, Fed Proc 42:264(Abstract), 1983.

9. Daniels CJ, Bole DG, Quay SC, Oxender DL: Proc Natl Acad Sci USA 78:5396-5400, 1981.

10. Landick R, Oxender DL: In Fleischer S, Fleischer B (eds): "Methods in Enzymology: Biomembranes," Vol. 97. New York: Academic Press, 1983, pp 146-153.

11. Landick R, Duncan JR, Copeland BR, Nazos PM, Oxender DL: J Cell Biochem (this issue).

12. Randall LL, Hardy SJS: Eur J Biochem 75:43-53, 1977.

13. Zwizinski C, Wickner W: J Biol Chem 255:7973-7977, 1980.

14. Wolfe PB, Silver P, Wickner W: J Biol Chem 257:7898-7902, 1982.

15. Date T, Zwizinski C, Ludmerer S, Wickner W: Proc Natl Acad Sci USA 77:827-831, 1980.

16. Date T, Goodman JM, Wickner W: Proc Natl Acad Sci USA 77:4669. 4673, 1980.

17. Enequist HG, Hirst TR, Hardy SIS, Harayama S, Randall LL: Eur J Biochem 116:227-233, 1981.

18. Josefsson LG, Randall LL: J Biol Chem 256:2504-2507, 1981.

19. Watts C, Silver P, Wickner W: Cell 25:347-353, 1981.

20. Ohno-Iwashita Y, Wickner W: J Biol Chem 258:1895-1900, 1983.

21. Walter P, Ibrahimi I, Blobel G: J Cell Biol 91:545-550, 1981.

22. Walter P, Blobel G: J Cell Biol 91:551-556, 1981.

23. Walter P, Blobel G: I Cell Biol 91:557-561, 1981.

24. Emr SD, Bassford PJ: J Biol Chem 257:5852-5860, 1982.

25. Emr SD, Hanley-Way S, Silhavy TJ: Cell 23:79-88, 1981.

26. Oliver DB, Beckwith J: Cell 25:765-772, 1981.

27. Oliver DB, Beckwith J: Cell 30:311-319, 1982.

28. Randall LL: Cell 33:231-240, 1983.

29. DiRienzo JM, Nakamura K, Inouye M: Annu Rev Biochem 47:481-532, 1978.

30. Steiner DF, Quinn PS, Chan SJ, Marsh J, Tager HS: Ann NY Acad Sci 343:1-16, 1980.

31. Parsegian A: Nature 221:844-846, 1969.

32. Wickner W: Annu Rev Biochem 48:23 45, 1979.

33. Chou PY, Fasman GD: Annu Rev Biochem 47:251-276, 1978.

34. von Heigne G, Blomberg C: Eur J Biochem 97:175-181, 1979.

35. Engelman DM, Steitz TA: Cell 23:411-422, 1981.

36. Hol WGJ, Halie LM, Sander C: Nature 294:532-536, 1981.

37. Bockris JO'M, Reddy AKN: "Modern Electrochemistry," Vol. 1. New York: Plenum Press, 1970, pp 48-72.

38. Chothia C: Nature 248:338-339, 1974. 